\title{
Fluxon propagation in long Josephson junctions with external magnetic field
}

\author{
Olsen, O.H.; Samuelsen, Mogens Rugholm
}

Published in:

Journal of Applied Physics

Link to article, DOI:

10.1063/1.328567

Publication date:

1981

Document Version

Publisher's PDF, also known as Version of record

Link back to DTU Orbit

Citation (APA):

Olsen, O. H., \& Samuelsen, M. R. (1981). Fluxon propagation in long Josephson junctions with external magnetic field. Journal of Applied Physics, 52(10), 6247-6251. https://doi.org/10.1063/1.328567

\section{General rights}

Copyright and moral rights for the publications made accessible in the public portal are retained by the authors and/or other copyright owners and it is a condition of accessing publications that users recognise and abide by the legal requirements associated with these rights.

- Users may download and print one copy of any publication from the public portal for the purpose of private study or research.

- You may not further distribute the material or use it for any profit-making activity or commercial gain

- You may freely distribute the URL identifying the publication in the public portal

If you believe that this document breaches copyright please contact us providing details, and we will remove access to the work immediately and investigate your claim. 


\section{Fluxon propagation in long Josephson junctions with external magnetic field}

O. H. Olsen

NIRO Atomizer, $R \& D$ System and Design Division, Gladsaxevej, DK-2860 Soeborg, Denmark

M. R. Samuelsen

Physics Laboratory I, The Technical University of Denmark, DK-2800 Lyngby, Denmark

(Received 9 February 1981; accepted for publication 31 March 1981)

The reflection of a single fluxon propagating in a Josephson line cavity influenced by an external magnetic field is examined numerically. We find a single reflected fluxon, an antifluxon, collapse of the incident fluxon, fission into a higher number of antifluxons or fluxons, and formation of breatherlike waves depending on the velocity of the incident fluxon and the magnitude of the external magnetic field. Approximations based on energy analysis describing the border lines between regions of different processes are presented.

PACS numbers: 74.50. + r, 84.40.Mk

\section{INTRODUCTION}

Nonlinear solitary waves are currently being used in a remarkable variety of contexts in almost every area of physics. In particular the sine-Gordon $2 \pi$-kink solution is ubiquitous in its application as a model for, e.g., dislocations in crystals, domain walls in ferromagnets, and propagation of flux quanta, fluxons, and in Josephson transmission lines.

The dynamics of fluxons on Josephson-junctions transmission lines has been studied extensively. In particular we mention the papers by McLaughlin and Scott ${ }^{1}$ and Kaup and Newell, ${ }^{2}$ which contain many references. In Refs. 1 and 2 a perturbation theory for fluxons propagating on Josephson lines with bias, impurities, and losses is formulated. Some of the results from this theory were compared with numerical solutions by Christiansen and Olsen. ${ }^{3}$ Costabile $e t$ $a l .{ }^{4}$ have given an exact solution to the sine-Gordon equation without perturbing terms for a finite line with open-end boundary conditions. For a finite line with fixed end boundary conditions corresponding to a short-circuited line DeLeonardis et al. ${ }^{5}$ obtained exact solutions using the same method. A numerical investigation of the reflection of a single fluxon on a semi-infinite Josephson line at a passive or an active boundary has been performed by Christiansen and Olsen. ${ }^{6}$ They found a single reflected fluxon, an antifluxon, absorption of the incident fluxon, or fission into an arbitrary number of fluxons, depending on the boundary conditions.

Besides the $2 \pi$-kink solution the sine-Gordon equation possesses the breather solution which recently has attracted considerable interest. ${ }^{7-10}$ The breather can be considered as a bound state of a fluxon and an antifluxon. Furthermore fluxons, antifluxons, and breathers possess the remarkable soliton property. Unlike the fluxons and the antifluxons, the breather does not require an activation energy, because its rest energy can range from 0 to $2 E_{0}$, where $E_{0}$ is the rest energy of the fluxons or the antifluxons. Further, breathers have an oscillatory degree of freedom, which increases their physical potential. For a finite line Costabile et al. have also found exact breather solutions. ${ }^{1}$ In Ref. 12 the effect of a boundary on a breather was examined numerically. Depending on the boundary condition (modeling either a passive or an active termination) the breather was reflected into a breather of decreased or increased energy.
Fluxon propagation in long Josephson line cavities has been used to explain the so-called zero field steps (without external magnetic field) in the current-voltage characteristics. ${ }^{13,14}$ The $n$th zero field step corresponds to a situation in which $n$ fluxons are propagating back and forth along the junction, being reflected at the ends. Each reflection creates a voltage pulse. Thus a single fluxon on a Josephson line at length $l$ produces a microwave signal of frequency $u / 2 l$, where $u$ is the velocity of the fluxon. Recently microwave emission from long Josephson tunnel junctions dc-current biased on zero-field steps and Fiske steps has been measured ${ }^{15}$ (the Fiske steps appear when an external magnetic field is applied). It is remarkable that the even Fiske steps coincide with the zero field steps. The measurements of the radiation emitted from the long junctions biased on Fiske steps show exactly the same features as the radiation from zero field steps. Therefore, it was concluded that Fiske steps in long junctions are due to propagating fluxons.

In the present paper we consider the reflection of a single fluxon in a long Josephson line cavity at a boundary with a condition which models the influence of an external magnetic field. Depending on the velocity $(u)$ of the incident fluxon and the magnitude $(\beta)$ of the external magnetic field we find a single reflected fluxon, collapse of the incident fluxon; a single reflected antifluxon and fission into fluxons and antifluxons. Further, we observe breatherlike solutions as a result of the reflection.

The paper is structured as follows: In Sec. II we review the model and derive the boundary condition. Section III contains our numerical results. In Sec. IV we show the results in a $u-\beta$-plane and explain some results by means of an approximation based on energy considerations. Further, in Sec. $V$ we comment on the results.

\section{THE MODEL}

The motion of fluxons in the oxide barrier (and in the London penetration layers) in a long and narrow Josephson junction is given by the sine-Gordon equation

$$
\phi_{x x}-\phi_{t t}=\sin \phi,
$$

where $\phi(x, t)$ is the space and time dependent phase difference between the two superconducting films. The spatial variable 
$x$ is measured in units of the Josephson penetration depth $\lambda_{J}=\left(\hbar / \mu_{0} 2 e d j_{J}\right)^{1 / 2}$ and the time $t$ in units of the reciprocal plasma frequency $\omega_{p}^{-1}, \omega_{p}=\left(2 e j_{J} / \hbar C\right)^{1 / 2}$. Here $-e$ is the electronic charge, $2 \pi \hbar$ is Planck's constant, $\mu_{0}$ is the permeability of free space, $j_{j}$ is the maximal Josephson current density through the barrier, $d$ is the magnetic thickness of the barrier $\left(2 \lambda+t_{0}\right)$, and $C$ is the capacitance per unit area of the junction $\left(\epsilon_{0} \epsilon_{r} / t_{0}\right)$.

We have neglected losses, since the effect of losses in the presence of bias is to determine the velocity of the fluxons by power balance.'

The surface current density in the London penetration layer in one of the films is given by

$$
i_{x}=\lambda_{J} j_{J} \phi_{x}
$$

and therefore the magnetic field in the oxide layer is given by

$$
H_{y}=i_{x}=\lambda_{J} \dot{j}_{J} \phi_{x} \text {. }
$$

Applying an external magnetic field parallel to the barrier and perpendicular to the length of the junction corresponds to fixing the magnetic field at the two ends $(x=0$ and $x=l)$ of the junction to the value of the external field $H_{\mathrm{ext}}$,

$$
H_{y}(x=0)=H_{y}(x=l)=H_{\text {ext }} .
$$

We introduce the dimensionless external magnetic field $\beta=H_{\mathrm{ext}} / \lambda_{J} j_{J}$, leading to the boundary condition

$$
\phi_{x}(0, t)=\phi_{x}(l, t)=\beta
$$

as the effect of the external magnetic field.

In experiments ${ }^{15}$ with Fiske steps the value of $\beta$ is of the order one.

\section{NUMERICAL RESULTS}

In order to study the behavior of a single fluxon in a Josephson line cavity of dimensionless length $l$ we solve the initial value problem,

$$
\begin{aligned}
& \phi_{x x}-\phi_{u}=\sin \phi, \\
& \phi_{x}(0, t)=\phi_{x}(l, t)=\beta, \quad-2 \leqslant \beta \leqslant 2 \\
& \phi(x, 0)=F(x, 0), \\
& \phi_{l}(x, 0)=F_{t}(x, 0),
\end{aligned}
$$

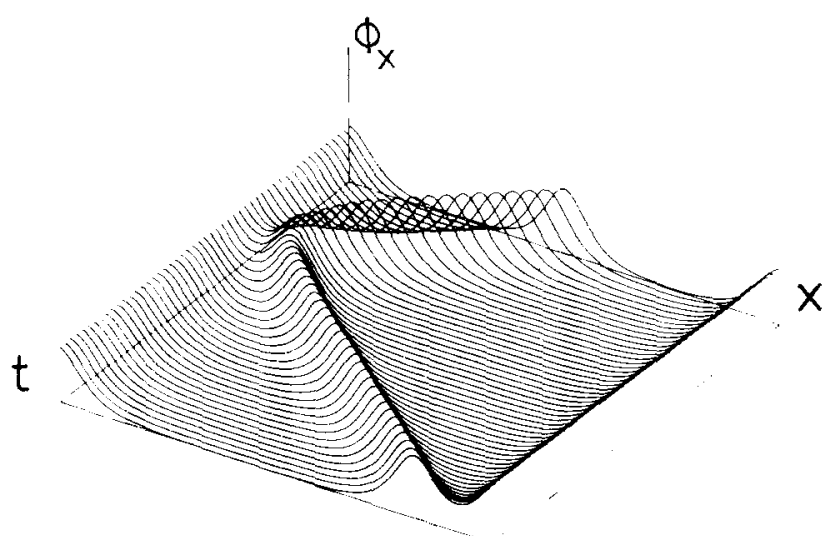

FIG. 1. Reflection of a single fluxon at the left end of a junction. Numerical solution of $\{3.1\}$ with $\beta=1.995, x_{0}=10, u=0.5$, and $0 \leqslant t \leqslant 50$. The results are displayed in terms of $\phi_{x}$ for $0 \leqslant x \leqslant 20$. The velocity of the reflected fluxon equals the velocity of the incident fluxon where the boundary conditions model the external magnetic field and the initial conditions are given by

$$
\begin{aligned}
& F(x, t)= 4 \tan ^{-1} \exp \left[\xi_{1}(\beta)+x\right] \\
&+4 \tan ^{-1} \exp \left[\gamma(u)\left(x+u t-x_{0}\right)\right] \\
&+4 \tan ^{-1} \exp \left[\xi_{2}(\beta)-l+x\right], \\
& \xi_{1}(\beta)=\left.\ln \{2 / \beta)\left[1+\left(1-\beta^{2} / 4\right)^{1 / 2}\right]\right\} \\
& \xi_{2}(\beta)= \ln \left\{(2 / \beta)\left[1-\left(1-\beta^{2} / 4\right)\right]^{1 / 2}\right\} \\
& \gamma(u)=1 /\left(1-u^{2}\right)^{1 / 2}
\end{aligned}
$$

Here the first and last terms in the expression for $F(x, t)$ represent parts of static fluxons such that the boundary conditions are satisfied and the energy in the cavity for $t=0$ is minimized. The middle term represents a fluxon with initial location and velocity $x_{0}$ and $u$, respectively. The fluxon is Lorentz contracted, $\gamma(u)$ is the Lorentz factor. Throughout this paper we have chosen $l=20$, corresponding to the size of a realistic junction. ${ }^{15}$

The numerical results are obtained by means of a computer program based on the method of characteristics and displayed in terms of $\phi_{x}(x, t)$.

Figure 1 shows the propagation of a single fluxon in a line cavity. The parameters in (3.1) are chosen to be $\beta=1.995, x_{0}=10, u=0.5$, and $0 \leqslant t \leqslant 50$. The fluxon is reflected into a fluxon with a velocity equal to the velocity of the incident fluxon. This event can be explained as a symmetric, three-soliton interaction, one static at $x \simeq 0$ and the other two propagating in opposite directions. The fluxon will then run back and forth along the junction being reflected at the ends. Similar results are obtained for $u=0.5, x_{0}$ $=10$, and $\beta>1.09$. The same qualitative behavior is found for $0 \leqslant u \leqslant 0.71$. In the next section we give a simple explanation based on energy considerations.

In Fig. 2 we show an incident fluxon which collapses because of the external magnetic field. The parameter values in (3.1) are chosen to be $\beta=0.8, x_{0}=10, u=0.5$, and $0 \leqslant t \leqslant 50$. As result of the collapse an oscillating mode starts, $\phi(0, t)$ oscillates from $2 \pi+4 \tan ^{-1} \exp \xi_{1}(\beta)$ to $2 \pi+4 \tan ^{-1} \exp \xi_{2}(\beta)$, corresponding to the values on a

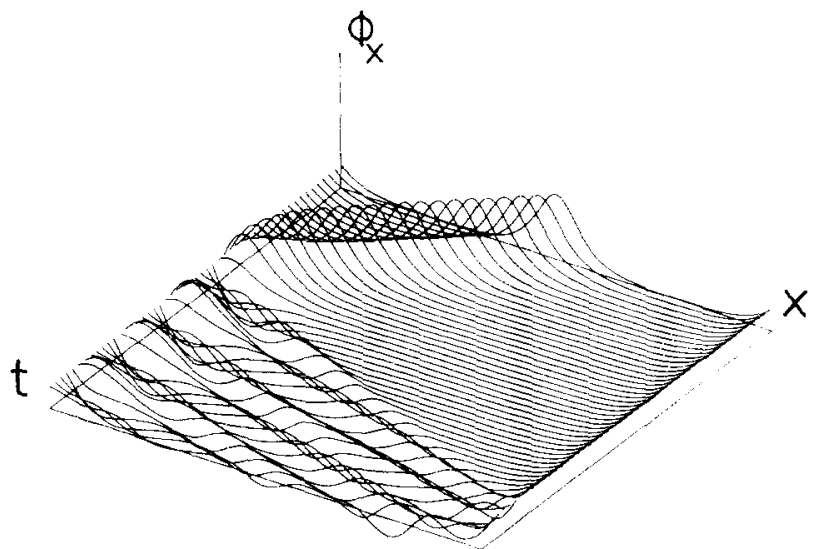

FIG, 2. Incident fluxon collapses as a result of the external magnetic field. Numerical solution of $(3.1)$ with $\beta=0.8, x_{t}=10, u=0.5$, and $0 \leqslant t \leqslant 50$. The results are displayed in terms of $\phi_{x}$ for $0 \leqslant x \leqslant 20$. After the collapse an oscillating mode starts. 


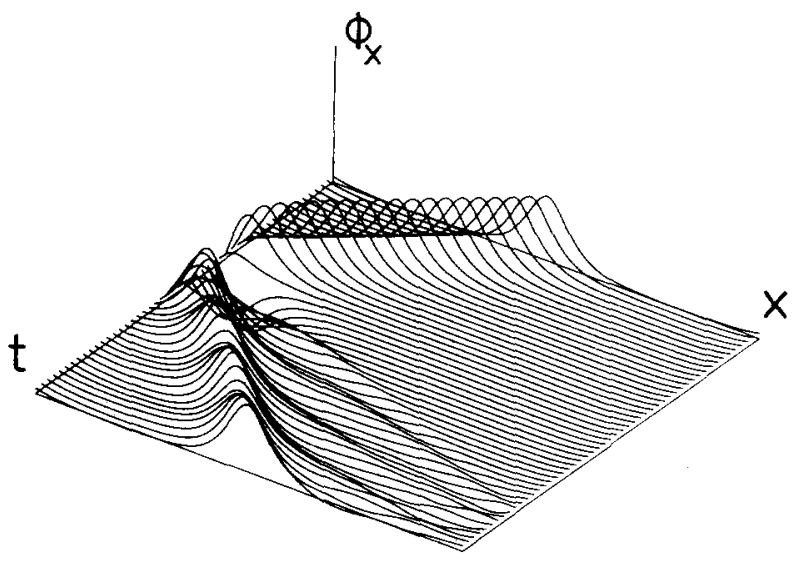

FIG. 3. Reflection of a single fluxon at the left end of a junction. Numerical solution of (3.1) with $\beta=0.2, x_{0}=10, u=0.5$, and $0 \leqslant t \leqslant 50$. The results are displayed in terms of $\phi_{x}$ for $0 \leqslant x \leqslant 20$. The reflected fluxon oscillates and produces radiation.

static fluxon where $\phi_{x}$ is equal to $\beta$.

Figure 3 shows the reflection of a fluxon at the left end of a line cavity. The parameter values in (3.1) are $\beta=0.2$, $x_{0}=10, u=0.5$, and $0 \leqslant t \leqslant 50$. The reflection event differs from the event in Fig. 1, i.e., a three-soliton interaction does not explain this phenomenon because the fluxon seems to be reflected into an antifluxon but eventually enters a fluxon mode. The velocity of the reflected fluxon is approximately equal to the velocity of the incident fluxon. Further, we remark the oscillatory behavior of the reflected fluxon resulting in radiation.

In Fig. 4 we show an incident fluxon which is converted into an almost static antifluxon. In this case the parameter values in (3.1) are chosen to be $\beta=0.096, x_{0}=10, u=0.5$, and $0 \leqslant t \leqslant 50$. The antifluxon either travels to the right at a small velocity or enters a fluxon mode traveling with a velocity which approximately equals the velocity of the incident fluxon (actually the velocity is smaller because of the radiation). A phaseplane analysis shows that no static antifluxon exists in this situation. The antifluxon in Fig. 4 is found to convert into a fluxon at $t \simeq 90$. For $\beta \lesssim 0.095$ and $u=0.5$ all

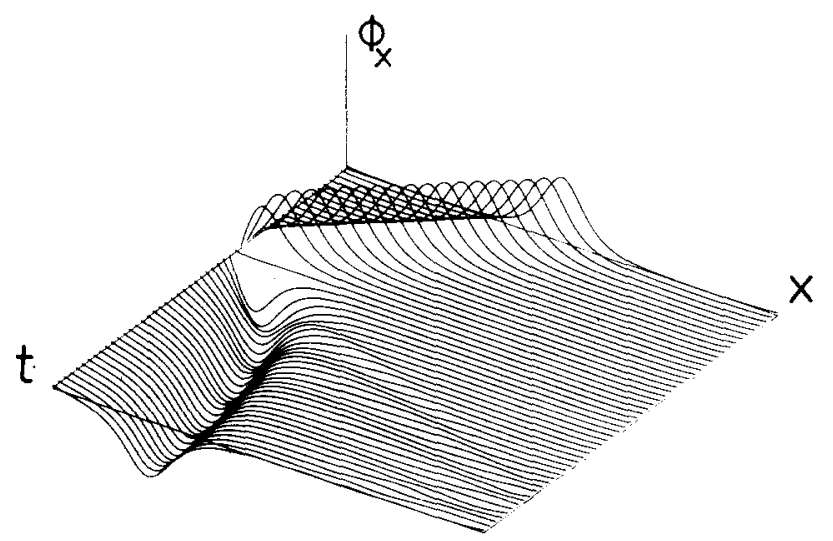

FIG. 4. Incident fluxon is reflected into an almost static antifluxon. Numerical solution of (3.1) with $\beta=0.096, x_{0}=10, u=0.5$, and $0 \leqslant t \leqslant 50$. The results are displayed in terms of $\phi_{x}$ for $0 \leqslant x \leqslant 20$. Eventually the antifluxon enters a fluxon mode.

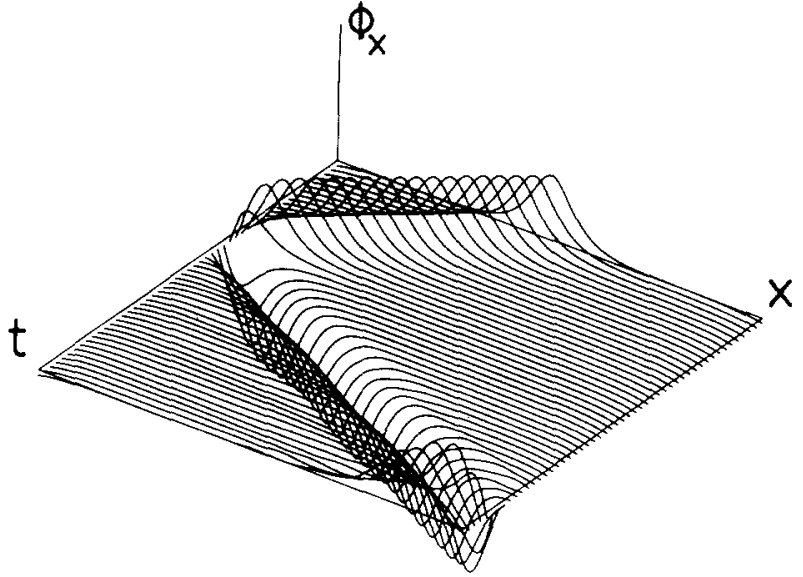

FIG. 5. Reflection of an incident fluxon into an amplified antifluxon. Numerical solution of (3.1) with $\beta=0.2, x_{0}=10, u=0.5$, and $0 \leqslant t \leqslant 50$. The results are displayed in terms of $\phi_{x}$ for $0 \leqslant x \leqslant 20$. Simulation of the first zero field step or the second Fiske step.

fluxons are found to be reflected into antifluxons. Again a simple energy analysis explains this behavior (see next section).

In Fig. 5 we show an incident fluxon which is reflected into an amplified antifluxon at the left end of a line cavity. The parameter values in (3.1) are $\beta=-0.2, x_{0}=10$, $u=0.5$, and $0 \leqslant t \leqslant 50$. At the right end the antifluxon is reflected into a reduced fluxon. Very little radiation is observed. This behavior continues ad infinitum and describes therefore the first zero field step or equivalently the second Fiske step (the value of $\phi$ at the boundaries increases $4 \pi$ as a result of a reflection). For $u=0.5$ and $-1.145 \leqslant \beta<0$ we find an amplified antifluxon, while for smaller values of $\beta$ the incident fluxon is fissioned into two or more antifluxons.

Finally, in this section we present results for incident fluxons at large velocities. In Fig. 6 the incident fluxon is reflected into three fluxons. The parameter values are $\beta=1.9, x_{0}=10, u=0.9$, and $0 \leqslant t \leqslant 60$. When the fluxons reach the right end of the cavity radiation is observed.

In Fig. 7 the incident fluxon is reflected into a breatherlike wave. The parameter values are $\beta=1.02, x_{0}=10$,

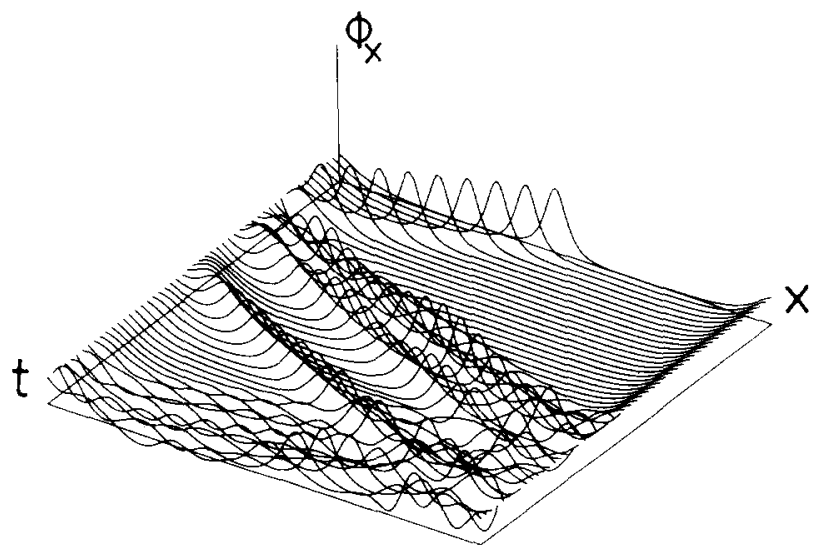

FIG. 6. Incident fluxon is fissioned into three fluxons. Numerical solution of $(3.1)$ with $\beta=1.9, x_{0}=10, u=0.9$, and $0 \leqslant t \leqslant 60$. The results are displayed in terms of $\phi_{x}$ for $0 \leqslant x \leqslant 20$. 


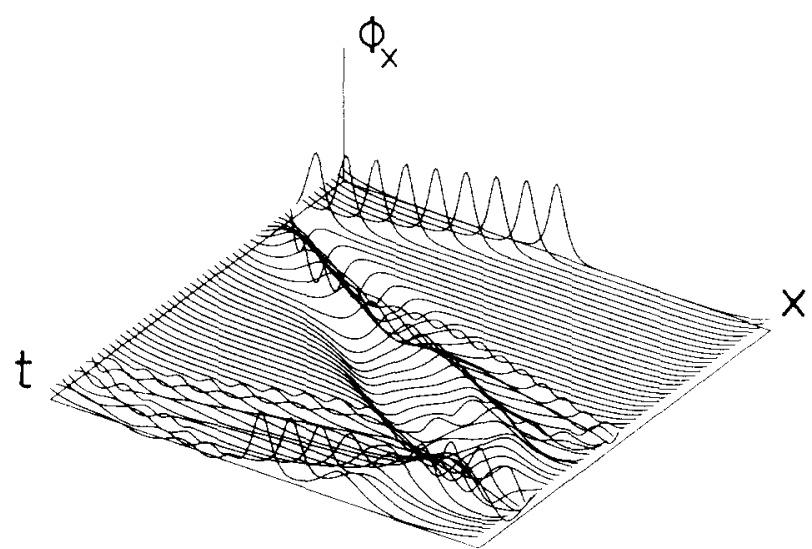

FIG. 7. As a result of the reflection, a breatherlike wave is formed. Numer:cal solution of $(3.1)$ with $\beta=1.02, x_{0}=10, u=0.95$, and $0 \leqslant t \leqslant 60$. At the right end the breather is divided into two fluxons.

$u=0.95$, and $0 \leqslant t \leqslant 60$. At the right end the breather is reflected as two fluxons. Thus at the left end $\phi$ increases $2 \pi$ while at the right end the increase is $4 \pi$.

In Fig. 8 we have shown a $u-\beta$ diagram, where $u$ is the velocity of the incident fluxon and $\beta$ is the magnitude of the external magnetic field. The figure illustrates the regions in which one of five processes take place: reflection of the incident fluxon into a fluxon (region I, illustrated in Figs. 1 and 3 ), collapse of the incident fluxon (region II, illustrated in Fig. 2), reflection of the incident fluxon into an antifluxon which is either reduced $(\beta>0)$ or amplified $(\beta<0)$ (region III, illustrated in Fig. 4 for $\beta<0$ ), fission of the incident fluxon into more than one antifluxon (region IV), and finally conversion of the incident fluxon into breatherlike waves or fission into a higher number of fluxons (region $\mathrm{V}$, illustrated in Figs 6 and 7). The full border lines between the regions are determined numerically, while the dot-dashed border line illustrates region $\mathrm{V}$, where some highly nonlinear phenomena take place. In the next section the border lines will be discussed.

\section{ENERGY ANALYSIS}

For the finite Josephson line we may introduce the dimensionless Hamiltonian

$$
H(t)=\int_{0}^{1}\left(\frac{1}{2} \phi_{x}^{2}+\frac{1}{2} \phi_{t}^{2}+1-\cos \phi\right) d x .
$$

Integrating by parts we get

$$
\begin{aligned}
\frac{d H}{d t}= & \int_{0}^{l} \phi_{t}\left(\phi_{l t}-\phi_{x x} \sin \phi\right) d x \\
& -\phi_{x}(0, t) \phi_{t}(0, t)+\phi_{x}(l, t) \phi_{t}(l, t) \\
= & -\beta \phi_{t}(0, t)+\beta \phi_{t}(l, t) .
\end{aligned}
$$

Thus the rate of change of the total energy of the system is equal to the effect absorbed or delivered by the external magnetic field. When $\beta=0$ the total energy is constant.

The energies inside the junction of the static fluxons placed at the boundaries can easily be found [insertion of, e.g., the left term in the expression for $F(x, t),(3.2)$ in (4.1)]

$$
H_{01}=4\left\{1-\left[1-(\beta / 2)^{2}\right]^{1 / 2}\right\} \text {. }
$$

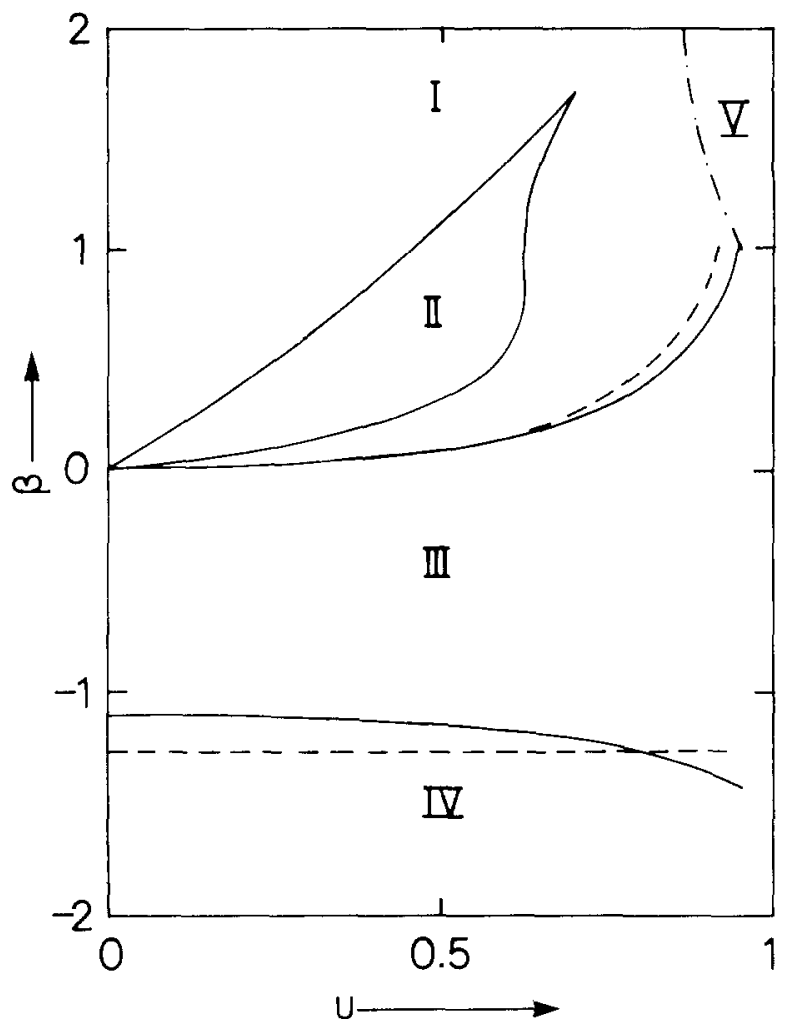

FIG. 8. Incident fluxon with the initial velocity $u$ strikes the boundary at $x=0$ with the boundary condition $\phi_{x}(0, t)=\beta$. The resulting process depends on $u$ and $\beta$, as illustrated. In region I the fluxon is reflected into a fluxon, in region II the fluxon collapses, in region III the fluxon is reflected into a single antifluxon, in region IV the fluxon is fissioned into antifluxons, while in region $\mathrm{V}$ the fluxon is fissioned into fiuxons or forms breatherlike waves. The full curves are numerically determined, the dashed curves are obtained by energy analysis, and the dot-dashed curve shows the border line for region $\mathrm{V}$.

Further we define

$$
H_{02}=8-H_{01}=4\left\{1+\left[1-(\beta / 2)^{2}\right]^{1 / 2}\right\}
$$

as the difference between the energy of a static fluxon and $H_{01}$. The energy of the incident fluxon is given by

$$
H_{f}=8 \gamma(u) \text {. }
$$

When we only consider the reflection at the left end of the cavity the change of energy is given by

$$
\Delta H=-\left.\beta \Delta \phi\right|_{x=0} .
$$

In order to explain the behavior of the upper border line between regions I and II in Fig. 8 we note that

$$
\begin{aligned}
\left.\Delta \phi\right|_{x=0} & =8 \tan ^{-1}\left((2 / \beta)\left\{1+\left[1-(\beta / 2)^{2}\right]^{1 / 2}\right\}\right) \\
& =-4 \sin ^{-1}(\beta / 2)
\end{aligned}
$$

corresponds to a change from a static fluxon of energy $H_{01}$ a static fluxon of energy $H_{02}$, both satisfying the boundary condition $\left.\phi_{x}\right|_{x=o}=\beta$. A condition that the fluxon survives the reflection at $x=0$ (and is reflected into a fluxon) can then be formulated,

$$
\Delta H=-\left.\beta \Delta \phi\right|_{x=0} \leqslant H_{\mathrm{inc}}-H_{02}=H_{f}+H_{01}-H_{02} .
$$

Insertion of $(4.3)-(4.5)$ into (4.7) yields

$$
\gamma(u) \leqslant \gamma\left(u_{1}\right)=g(\beta),
$$


where

$$
g(\beta)=\left[1-(\beta / 2)^{2}\right]^{1 / 2}+(\beta / 2) \sin ^{-1}(\beta / 2) .
$$

Here $u=u_{1}(\beta)$ yields a theoretical determination of the upper border line between regions I and II. The numerically determined border line differs less than $1 \%$ from the theoretical (4.8). The expression (4.8), however, does not explain the intersection point between the upper and lower border lines between regions I and II.

A somewhat similar analysis can be applied to explain the border line between regions I and III, i.e., the border line between regions where a fluxon is reflected into a fluxon and a fluxon is reflected into an antifluxon. If the incident fluxon is reflected into an antifluxon at the left end of the line $\phi$ changes $-4 \pi$. After the reflection the energy must exceed the energy of a static antifluxon (i.e., 8). A condition for reflection of a fluxon into an antifluxon can then be formulated,

$$
H_{f}>\Delta H+8=4 \pi \beta+8,
$$

or after rearranging,

$$
\begin{aligned}
& u \geqslant u_{2}(\beta) \\
& =\{\pi \beta[1+(\pi / 4) \beta]\}^{1 / 2} /[1+(\pi / 2) \beta] \quad \text { for } \beta \geqslant 0 .
\end{aligned}
$$

The condition (4.9a) is trivially fulfilled for $\beta \leqslant 0$ (for $\beta=0$ the situation corresponds to the well known fluxon-antifluxon solution). The expression $u=u_{2}(\beta)$ yields a theoretical determination of the border line. In Fig. 8 the function $u_{2}(\beta)$ is shown as the dashed curve. A good agreement is observed for $\beta<0.2$. For increasing values of $\beta$ the deviation between the full and the dashed curve increases. This is due to radiation (i.e., energy dispersion), which is observed at large velocities of the incident fluxon; therefore, the analysis above fails.

An explanation of the border line between regions III and IV, i.e., the border line between regions where a fluxon is reflected into a single fluxon and a fluxon is reflected into antifluxons, has not been found. We have, however, found a simple approximation to the border line. An expression for the velocity of the reflected antifluxon can be written down, namely,

$$
H_{\text {reff }}=8 \gamma\left(u_{\text {reff }}\right)=8 \gamma(u)-4 \pi \beta,
$$

where $H_{\text {reff }}$ is the energy of the resulting antifluxon propagating with the velocity $u_{\text {refl }}$. After rearranging we get

$$
u_{\mathrm{refl}}=\left\{1-\frac{1}{[\gamma(u)-(\pi / 2) \beta]^{2}}\right\}^{1 / 2},
$$

which is in good agreement with the results obtained from numerical experiments for $\beta>-0.5$. If fission takes place the following condition must be fulfilled:

$$
-4 \pi \beta \geqslant 8 \text {, }
$$

stating that the energy delivered by the external magnetic field exceed the energy of a static antifluxon. Thus, a simple approximation to the border line is $\beta=-4 / \pi \simeq-1.27$. In Fig. 8 the dashed line corresponds to this simple approximation. A better approximation would require a detailed understanding of the fission process.
The border line for region $\mathrm{V}$ (the dot-dashed curve in Fig. 8 ) is only qualitative correct. In this region some highly nonlinear processes take place which we have not been able to explain by means of energy analysis approximation. This region, however, deserves a detailed examination because it is likely to find the mechanism of the Fiske steps in this region (see, e.g., Fig. 7). In particular the first Fiske step may be generated by a mode in which a fluxon propagates (e.g., to the left). At the left end of the line the fluxon collapses and a part of its energy is reflected in the form of a breather, which in turn launches a new fluxon at the right end.

Finally, we remark that the lower border line between regions I and II still remains to be explained.

\section{CONCLUSION}

In the present paper we have examined the influence of an external magnetic field on a propagating fluxon. The external magnetic field is modeled by a certain boundary condition.

Depending on the magnitude of the external magnetic field and the velocity of the incident fluxon, we find (Fig. 8) that the fluxon either is reflected into a fluxon (region I), collapses (region II), is reflected into an antifluxon (region III), is fissioned into antifluxons (region IV) and fluxons or forms breatherlike waves (region V). Satisfactory explanations based on energy analysis have been found for the upper border line between regions I and II and for the border line between regions II and III. A simple approximation to the border line between regions III and IV is also given. The lower border line between regions II and III still remains to be explained. Furthermore, region $V$ needs a detailed investigation because the mechanism of the Fiske steps are to be found here.

The results described for a fluxon also hold for an antifluxon if $\beta$ is replaced by $-\beta$.

A more realistic model appears if loss and bias are taken into account in the sine-Gordon equation. Thus, the first and second Fiske steps and the first zero field step (which only require the presence of a single fluxon) have been simulated. ${ }^{16}$

'D. W. McLaughlin and A. C. Scott, Phys. Rev. A 18, 1652 (1978). ${ }^{2}$ D. J. Kaup and A. C. Newell, Proc. R. Soc. (London) Ser. A 361, 413 (1978).

${ }^{3}$ P. L. Christiansen and O. H. Olsen, Wave Motion 2, 185 (1980).

${ }^{4}$ G. Costabile, R. D. Parmentier, B. Savo, D. W. McLaughlin, and A. C. Scott, Appl. Phys. Lett. 32, 5877 (1978).

${ }^{5}$ R. M. DeLeonardis, S. E. Trullinger, and R. F. Wallis, J. Appl. Phys. 51, $1211(1980)$.

${ }^{6}$ P. L. Christiansen and O. H. Olsen, Physica D 10, 412 (1980).

${ }^{7}$ M. Inoue and S. G. Chung, J. Phys. Soc. Jpn. 46, 1594 (1979).

${ }^{8}$ E. Stoll, T. Schneider, and A. R. Bishop, Phys. Rev. Lett. 47, 937 (1979).

${ }^{9}$ A. R. Bishop, Z. Phys. B 37, 357 (1980).

${ }^{10}$ A. C. Scott, Phys. Scripta 20, $509(1979)$.

${ }^{11}$ C. Costabile, R. D. Parmentier, and B. Savo, J. Phys. (Paris) 39, 567 (1978).

${ }^{12}$ O. H. Olsen and M. R. Samuelsen, J. Appl. Phys. 52, 2913 (1981).

${ }^{13}$ S. N. Erné and R. D. Parmentier, J. Appl. Phys. 51, 5025 (1980).

${ }^{14}$ T. A. Fulton and R. C. Dynes, Solid State Commun. 12, 57 (1973).

${ }^{15}$ B. Dueholm, O. A. Levring, J. Mygind, N. F. Pedersen, O. H. Soerensen, and M. Cirillo Phys. Rev. Lett. (in press).

${ }^{16}$ B.Dueholm, O. A. Levring, J. Mygind, M. R. Samueisen, O. H. Olsen, and M. Cirillo (to be published). 